\title{
Análise de Resíduos de Pesticidas em Ar ATMOSFÉRICO
}

Luciane Gonçalves dos Santos* Eliana Freire Gaspar de Carvalho Dores** Carolina Lourencetti***

\section{Resumo}

A determinação dos níveis e a distribuição de pesticidas na atmosfera podem contribuir para o melhor entendimento do risco causado por essas substâncias à saúde humana. Considerando a necessidade de procedimentos eficientes e confiáveis para a determinação dos pesticidas nessa matriz, este trabalho apresenta uma breve discussão sobre procedimentos utilizados para a amostragem, amostradores ativos e passivos, e análise de pesticidas no ar, assim como procedimentos que vêm sendo utilizados na validação dos mesmos. Embora os estudos demonstrem um esforço analítico para a obtenção de dados confiáveis, novos estudos são requeridos para aprimorar os procedimentos de amostragem e métodos de análise de pesticidas em ar atmosférico.

Palavras-chave: Pesticidas; Ar atmosférico; Procedimentos de Amostragem e Análise.

\section{INTRODUÇÃO}

O rápido crescimento da agricultura nos últimos anos inseriu o uso diversificado e intensivo de pesticidas, com a finalidade de aumentar a produtividade e atender à demanda mundial de alimentos.

À medida que essas substâncias são aplicadas nas lavouras, podem sofrer diferentes processos de dispersão, transformação e transporte e atingir os diferentes compartimentos ambientais, como a atmosfera, o solo, as águas superficiais e subterrâneas, podendo causar problema de intoxicação pela ingestão gradativa desses produtos presentes na água potável e em alimentos. É importante ressaltar que os

\footnotetext{
*Discente do Programa de Pós-Graduação em Recursos Hídricos - Universidade Federal de Mato Grosso, Cuiabá. E-mail: lucianegsm@yahoo.com.br.

**Docente e Pesquisadora do Programa de Pós-Graduação em Recursos Hídricos - UFMT. E-mail: eliana@ufmt.br.

***Bolsista PRODOC, Pesquisadora do Programa de Pós Graduação em Recursos Hídricos - UFMT. Email: carollourencetti@yahoo.com.br.
} 
impactos causados à saúde humana e aos recursos naturais pelos diferentes pesticidas com atual registro para uso no Brasile em outros países são ainda pouco conhecidos.

Estudos de monitoramento ambiental, realizados por meio de pesquisas de campo bem planejadas, são apontados, por diversos autores, como o melhor procedimento de avaliação e, consequentemente, minimização da contaminação por pesticidas (COHEN et al., 1995; ALBANIS et al., 1998; AZEVEDO et al., 2000; FILIZOLA et al., 2002). Embora esses estudos apresentem desvantagens, como a necessidade de métodos analíticos multirresíduos eficientes e capazes de detecção de baixos valores de concentração, elevado custo e consumo de tempo, devido ao grande número de análises que devem ser realizadas (SPADOTTO et al., 2004), os mesmos fornecem resultados da situação do local, como informações sobre níveis de concentração e distribuição dos pesticidas no ambiente.

A avaliação da distribuição de pesticidas na atmosfera é de fundamental importância, pois este é um meio de movimentação dos pesticidas para regiões distantes da área de aplicação. Uma vez realizada a avaliação, essas informações poderão subsidiar políticas de regulamentação desses compostos e ações mitigadoras para danos ambientais.

Considerando os aspectos expostos, este trabalho aborda os pesticidas e procedimentos de determinação dos mesmos no ar, assim como compara níveis de diferentes países.

\section{DEFinição e ClaSSIFICAÇÃo de PESTICIDAS}

A denominação dada ao grupo de substâncias químicas destinadas ao controle de pragas (animais ou vegetais) e de doenças de plantas varia muito. No Brasil, essas substâncias são classificadas como praguicidas, defensivos agrícolas, pesticidas e, mais recentemente, agrotóxicos (RANGEL, 2008). Esta última nomenclatura somente foi adotada após a sanção da Lei Federal n. ${ }^{\circ} 7.802$, de 11 de julho de 1989 (BRASIL, 1989), alterada pela Lei n. ${ }^{\circ}$ 9.974, de 06 de junho de 2000 (BRASIL, 2000), atualmente regulamentada pelo Decreto n. ${ }^{\circ} 4.074$, de 04 de janeiro de 2002 (BRASIL, 2002), modificado pelos Decretos n. ${ }^{\circ} 5.981$, de 06 de dezembro de 2006 (BRASIL, 2006), e n. 5.549, de 29 de setembro de 2005 (BRASIL, 2005), que define agrotóxicos como produtos e agentes de processos físicos, químicos ou biológicos destinados ao uso nos setores de produção, no armazenamento e beneficiamento de produtos agrícolas, nas pastagens, na proteção de florestas, nativas ou implantadas, e de outros ecossistemas e também de ambientes urbanos, hídricos e industriais, cuja finalidade seja alterar a composição da flora ou da fauna, a fim de preservá-las da ação danosa de seres vivos considerados nocivos; e ainda como substâncias e produtos empregados como desfolhantes, dessecantes, estimuladores 
e inibidores de crescimento. Considerando que o termo pesticida é o mais empregado no meio científico, o mesmo foi adotado neste trabalho.

Os pesticidas são classificados pela estrutura química e pelo organismo alvo. De acordo com o organismo alvo se classificam em: inseticidas, fungicidas, herbicidas, acaricidas, rodenticidas, carrapaticidas, entre outros. Em relação à estrutura química, podem ser classificados em organofosforados, carbamatos, organofosforados, piretróides, organonitrogenados, triazinas, benzimidazóis, triazol, acetanilina, dentre outros. Cada classe química apresenta um radical comum que atribui, a todos os compostos derivados, sua capacidade de ação inseticida, herbicida ou outra qualquer.

A ampla e complexa diversidade entre as moléculas de pesticidas confere às mesmas distintas propriedades físicas e químicas, resultando assim em diferente potencial toxicológico e comportamento no ambiente (ARMAS et al., 2007).

\section{DinÂMICA DOS PESTICIDAS NO AMBIENTE - PRESENÇA NO AR}

A distribuição ambiental dos pesticidas é influenciada por suas propriedades físicas e químicas (como volatilidade, viscosidade, solubilidade em água e pressão de vapor), condições meteorológicas (direção e velocidade do vento, temperatura, umidade relativa, estabilidade atmosférica, entre outras) e manejo (HAPEMAN et al., 2003).

De uma forma geral, a aplicação dos pesticidas dá-se por pulverização aérea ou terrestre, podendo ser manual ou mecânica. Durante a aplicação, cerca de $30 \%$ a $50 \%$ da quantidade aplicada pode ser perdida para a atmosfera por deriva (VAN DEN BERG et al., 1999). Após a aplicação e deposição no solo, distribuemse pelas fases gasosa, aquosa e sólida (material orgânico e inorgânico) dessa matriz. Pelos processos de escoamento superficial e lixiviação, podem atingir os recursos hídricos superficiais e subterrâneos, respectivamente. Dependendo das concentrações nos vários compartimentos, das propriedades físicas e químicas dos pesticidas e das condições climáticas, os mesmos podem se volatilizar e ser transportados pela atmosfera (VAN JAARSVED, VAN PUL, 1999; MILLET et al., 1996; DOBSON et al., 2006; FERRARI et al., 2003). Embora os pesticidas presentes no solo possam sofrer diferentes fenômenos, como a erosão hídrica ou erosão eólica, sendo adsorvidos em pequenas partículas do solo transportadas pelo vento, a intensidade desses processos é baixa, prevalecendo assim a volatilização como o principal fator contribuinte para a entrada de pesticidas na atmosfera (DOBSON et al., 2006).

Diversos fatores são apresentados como importantes na emissão e transporte de pesticidas na atmosfera e áreas adjacentes aos locais de aplicação, como técnicas de pulverização e fatores ambientais (GIL, SINFORT, 2005). Outros parâmetros, 
como quantidade utilizada, formulação, tipo de cultura, característica do solo e propriedades físicas e químicas dos pesticidas também são citados (VAN DJIK, 1999; LEPRI, 1996). Devido à possibilidade de ocorrência de difusão para áreas distantes do local de aplicação, como zonas urbanas, problemas de poluição atmosférica por pesticidas não ficam localizados somente em regiões agrícolas (SCHEYER et al., 2007).

$\mathrm{Na}$ atmosfera, os pesticidas podem ser encontrados distribuídos nas fases gasosas, aquosa ou sólida (particulado) (SCHEYER et al., 2005; MILLET et al., 1997), o que afeta o transporte e o destino dos pesticidas na atmosfera (DOBSON et al., 2006). A taxa de remoção desses agentes da atmosfera é dependente da reatividade química e fotoquímica, bem como dos processos de deposição seca (gás e partícula) e úmida (precipitação) (VAN PULL et al., 1999). De acordo com Briand et al. (2002), as propriedades físicas e químicas das moléculas que mais influenciam na distribuição dos pesticidas nas fases gasosa e particulada são a pressão de vapor, a constante de Henry e a persistência. Valores altos dessas três propriedades indicam elevado potencial para atingir o compartimento atmosférico (EMPRESA BRASILEIRADE PESQUISAAGROPECUÁRIA, 2005).

\section{DETERMINAÇÃo DE PESTICIDAS NA ATMOSFERA}

A determinação de pesticidas nas matrizes ambientais (água, solo, ar e plantas alvo) e em alimentos é apontada como o ponto de partida para o estabelecimento de processos que avaliam o risco potencial desses compostos para o ambiente e para a saúde humana (FALCONER, 2002; VILLA, MC LEOD, 2002).

$O$ primeiro relato da presença de pesticidas na atmosfera foi apresentado no final da década de 1950, quando havia significante uso dos pesticidas clorados, a primeira geração de pesticidas, como DDT, lindano e dieldrin (UNSWORTH et al., 1999). Os poluentes orgânicos persistentes (POPs) são os compostos mais investigados na matriz ar, devido à elevada persistência e volatilidade (AULAGNIER et al., 2008; BAKER et al., 1996; DUYZER, 2003; JAWARD et al., 2004; LEPRI et al., 1995; HERCEG et al., 2003). Apresença de pesticidas de elevado uso atual também vem sendo estudada nessa matriz.

Os níveis de pesticidas no ar são geralmente baixos; assim, técnicas de amostragem, pré-concentração e extração apropriadas são necessárias para atingir a sensibilidade dos instrumentos analíticos (YUSÀ et al., 2009).

A amostragem de pesticidas em ar consiste na pré-concentração dos analitos em filtro e/ou adsorventes sólidos com o auxílio de equipamentos de coleta de ar de alto e baixo volumes (amostradores ativos) ou amostradores passivos (GIL et al., 2005), posterior extração dos analitos adsorvidos com técnicas de extração como Soxhlet 
e sonicação e identificação e quantificação por cromatografia gasosa, acoplada a detectores clássicos e espectrômetro de massas e, mais recentemente, devido à maior polaridade e baixa estabilidade térmica da nova geração de pesticidas, cromatografia líquida acoplada à espectrometria de massas (ALEGRIA et al., 2006; AULAGNIER et al., 2008; BOTTERMAN et al., 2008; LEWIS et al., 1982; FERRARI et al., 2003; MURAYAMA et al., 2000; YUSÀ et al., 2009). A Figura 1 apresenta de forma esquemática amostradores ativos e passivos.

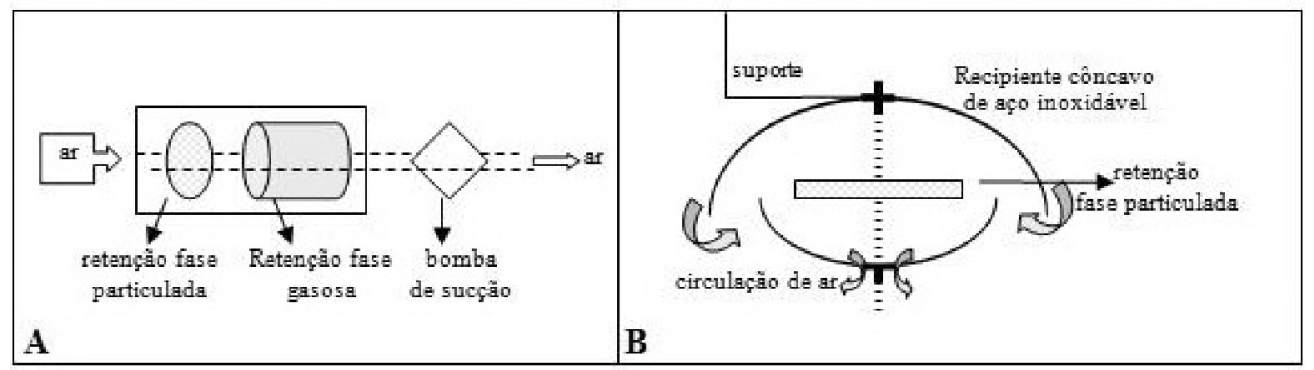

Figura 1. Esquema de amostradores ativos (A) e passivos (B). Modificado de YUSÀ et al., 2009.

Nos amostradores ativos, as substâncias químicas, pesticidas no caso desta discussão, presentes nas fases gasosa e particulada do ar, são coletadas e préconcentradas pela passagem forçada de ar por filtros e/ou adsorventes com auxílio de uma bomba de sucção. Os pesticidas presentes na fase particulada são retidos no filtro, e os presentes na fase gasosa, pelo adsorvente. Os amostradores ativos podem ser de alto e baixo volumes, dependendo da bomba de sucção utilizada. É importante mencionar que o tipo de amostrador, assim como as condições de operação, como o fluxo de ar, o adsorvente utilizado e o tempo de amostragem, estão estritamente relacionados com os propósitos do estudo. Os amostradores de alto volume são utilizados e recomendados para pré-concentração de pesticidas em ambientes abertos, onde a concentração dos mesmos é baixa, devido ao efeito de diluição e transporte dos mesmos pelo vento. Os amostradores de baixo volume também são utilizados para ambientes abertos, mas em razão do baixo volume de ar amostrado vêm sendo utilizados, principalmente, em avaliações de exposição ocupacional e estudos controlados em laboratório. Um documento com detalhada revisão da literatura (YUSÀ et al., 2009) apresenta trabalhos que empregaram amostradores de alto e baixo volumes com distintos adsorventes, tempo de coleta e fluxo de ar. Dos trabalhos descritos, os fluxos de trabalho das bombas variam de $0,35 \mathrm{~m}^{3} \mathrm{~L}^{-1}$ a $34 \mathrm{~m}^{3} \mathrm{~L}^{-1}$ e o tempo de coleta, de 5,5 horas a 168 horas. 
Os amostradores passivos são instrumentos que coletam os pesticidas da atmosfera sem o auxílio de uma bomba de sucção. Consiste de um material com alta capacidade de retenção dos analitos de interesse. Esse tipo de sistema permite a amostragem de ar em áreas onde a logística de uso de amostradores ativos por longos períodos não seria possível, como em locais remotos com ausência de suprimento de energia. Os amostradores passivos são capazes de coletar somente substâncias na fase gasosa do ar e a duração das amostragens varia entre semanas e meses, período mais longo do que o requerido quando são utilizados os amostradores ativos. Um dos grandes desafios do uso de amostradores passivos é estimar a concentração dos analitos em ar a partir das concentrações encontradas no material coletor contido no amostrador. Embora os amostradores passivos sejam menos confiáveis do que os amostradores ativos, os valores de concentração de pesticidas em ar encontrados pelos amostradores passivos são apontados como aproximadamente 2 a 3 vezes o valor da concentração determinada com os sistemas ativos (HARNER et al., 2006).

Tanto para os amostradores ativos como os passivos, é necessário um material capaz de reter os pesticidas durante a amostragem. Os pesticidas na fase particulada são retidos durante a amostragem ativa em filtros de fibra de vidro ou filtros de fibra de quartzo, cujos diâmetros variam entre $9 \mathrm{~cm}$ e $30 \mathrm{~cm}$, dependendo do amostrador utilizado. Com relação aos pesticidas presentes na fase gasosa, estes podem ser retidos em adsorventes, como a resina polimérica hidrofóbica Amberlita, XAD-2 (FONTANALS et al., 2007), PUF, espuma de poliuretano geralmente utilizada em colchões e travesseiros, e Tenax TA(BRIAND et al., 2002), resina com baixa área superficial e baixa afinidade por água. O XAD-2, considerado umadsorvente universal, é um dos mais utilizados, apresenta alta área superficial e alta eficiência na préconcentração de herbicidas, fungicidas e inseticidas.

A eficiência de diferentes tipos de adsorventes tem sido avaliada de forma isolada e em conjunto. Dobson et al. (2006) realizaram um estudo comparativo de alguns materiais. Os seguintes adsorventes e combinações dos mesmos foram comparados: XAD-2 com PUF, XAD-4 com PUF, XAD-2 com uma combinação ("sanduíches") PUF-XAD-2-PUF, e PUF com a combinação PUF-XAD-4-PUF. Segundo os autores, a eficiência de aprisionamento dos pesticidas nos "sanduíches" são maiores, seguido das resinas XAD-2 e XAD-4, e PUFs. No entanto, embora os "sanduíches" sejam ligeiramente melhores do que XAD-2, a utilização de XAD-2 é recomendada, porque os "sanduíches" são mais compactos, de forma que menos ar é bombeado e os adsorventes têm menos contato com o ar.

Uma relação de diferentes estudos apresentados na literatura sobre determinação de diversas classes de pesticidas em ar, descrevendo os procedimentos de 
amostragem, adsorvente, procedimento de extração e de quantificação discutidos neste trabalho, é apresentada na Tabela 1.

Similarmente aos estudos que identificam e quantificam pesticidas em matrizes ambientais como solo, água e sedimento, a determinação de pesticidas em ar também requer métodos analíticos simples, precisos e exatos. Esses parâmetros são considerados importantes em estudos mais complexos, como os de monitoramento ambiental, devido ao elevado número de análises requeridas (SPADOTTO et al., 2004). A confiabilidade dos resultados obtidos nesses estudos é também importante, uma vez que podem ser utilizados para estabelecer limites de qualidade do ar e em estudos de avaliação de risco humano (BAKER et al., 1996) e ambiental (DUYZER, 2003).

\section{VALIDAÇão de MÉTOdos de ANÁLISE de PESTICIDAS NA ATMOSFERA}

Para as matrizes solo, água e sedimento, o procedimento mais utilizado para a validação dos métodos analíticos é o estudo de recuperação utilizando amostras fortificadas ou material de referência certificado, quando disponíveis (RIBANI $e t$ $a l ., 2004)$. A validação de métodos para análise de pesticidas em ar ainda não é uma prática sistemática, e somente alguns estudos descritos na literatura apresentam dados sobre parâmetros de validação.

A avaliação da eficiência da extração dos analitos presentes no adsorvente utilizado durante a amostragem é o procedimento mais utilizado nesse tipo de estudo (ALEGRIA et al., 2006; AULAGNIER et al., 2008; BATTERMAN et al., 2008; FERRARI et al., 2003), sendo que alguns trabalhos avaliam possíveis perdas dos analitos retidos no adsorvente, passando ar pelo cartucho fortificado com os padrões dos pesticidas (BAKER et al., 1996; FERRARI et al., 2003). Embora haja uma preocupação com relação à eficiência da extração dos pesticidas retidos no adsorvente, a otimização e a avaliação da eficiência da coleta da amostra não vêm sendo investigadas.

\section{OCORRÊNCIA DE PESTICIDAS NA ATMOSFERA}

Os pesticidas presentes nas fases gasosa, particulada e líquida do ar podem oferecer risco à saúde humana e ao ambiente. Considerando as formas de deposição dos pesticidas da atmosfera, o homem está exposto àqueles presentes no ar por absorção dérmica, inalação e ingestão.

Estudos de avaliação da distribuição de pesticidas no ar são ainda escassos no Brasil. Como apresentado na Tabela 1, a quase totalidade dos trabalhos vem sendo realizada em países de clima temperado. De uma forma geral, nessas regiões as altas concentrações dos pesticidas no ar são dependentes da sazonalidade, ocorrendo 
nos meses da primavera e verão, coincidente com o período de aplicação e temperaturas mais elevadas.

Alguns trabalhos apresentados na literatura vêm tentando associar a presença de pesticidas encontrados em amostras de ar coletadas emáreas rural e urbana (MOTELAY et al., 2005; HERCEG et al., 2003) à sua ocorrência nas matrizes ar e água de chuva (AULAGNIER et al., 2008; DUYZER, 2003). Embora valores de concentrações mais elevadas venham sendo encontrados próximo aos pontos de aplicação (MAJEWSKI et al., 1998), diversos pesticidas têm sido detectados em locais distantes dos pontos de aplicação, como zonas urbanas e áreas remotas (Tabela 1).

\section{CONSIDERAÇões FINAIS}

Embora os estudos apresentados na literatura sobre pesticidas em ar demonstrem o esforço analítico para obtenção de dados confiáveis, novos trabalhos ainda são requeridos para aprimorar o procedimento de amostragem, de extração e determinação dos mesmos. Esses dados, além de fornecerem resultados que permitam um melhor entendimento da ocorrência e destino dos pesticidas de uso atual na atmosfera, poderão ser utilizados para estudos mais complexos que avaliem o risco humano de exposição a eles, considerando que a exposição inalatória é uma importante via de entrada dessas substâncias no organismo.

\section{REFERÊNCIAS}

ALBANIS, T. A.; HELA, D. G.; SAKELLARIDES, T. M.; KONSTANTINOU, I. K. Monitoring of pesticide residues and their metabolites in surface and underground waters of Imathia (N. Greece) by means of solid-phase extraction disk and gas chromatography. Journal of Chromatography, A, Amsterdam; New York, v. 823, n. 1-2, p. 59-71, Oct., 1998.

ALEGRIA, H.; BIDLEMAN, T. F.; SHAW, T. J. Organochlorine pesticides in ambient air of Belize, Central America. Environmental Science and Technology, Washington, v. 34, n. 10, p. 1953-1958, May, 2000.

ALEGRIA, H.; BIDLEMAN, T. F; FIGUEROA, M. S. Organochlorine pesticides in the ambient air of Chiapas, Mexico. Environmental Pollution, Barking, v. 140, n. 3, p. 483-491, Apr., 2006.

ARMAS, E. D.; MONTEIRO, R. T. R.;ANTUNES, P. M.; SANTOS, M. A. P. F; CAMARGO, P. B.;ABAKERLI, R. B. Diagnóstico espaço-temporal da ocorrência de 
herbicidas nas águas superficiais e sedimentos do rio Corumbataí e principais afluentes. Quimica Nova, São Paulo, v. 30, n. 5, p. 1119-1127, Set/Out, 2007.

AULAGNIER, F.; POISSANT, L.; BRUNET, D.; BEAUVAIS, C.; PILOTE, M.; DEBLOIS, C.; DASSYLVA, N. Pesticides measured in air and precipitation in the Yamaska Basin (Québec): Occurrence and concentrations in 2004. The Science of the total Environmental, Amsterdam, v. 394, n. 2, p. 338-348, May, 2008.

AZEVEDO, D. A.; LACORTE, S.; VINHAS, T.; VIANA, P.; BARCELÓ, D.

Monitoring of pesticides and other organic pollutants in river water from Portugal by gas chromatography-mass spectrometry and liquid chromatography-atmospheric pressure chemical ionization mass spectrometry. Journal of Chromatography, A, Amsterdam; New York, v. 879, n. 1, p. 13-26, May, 2000.

BAILEY, R.; BELZER, W. Large volume cold on-column injection for gas chromatography-negative chemical ionization-mass spectrometry analysis of selected pesticides in air samples. Journal of Agricultural and Food Chemistry, Easton, v. 55, n. 4, p. 1150-1155, Feb., 2007.

BAKER, L. W.; FITZELL, D. L.; SEIBER, J. N.; PARKER, T. R.; SHIBAMOTO, T.; POORE, M. W.; LONGLEY, K. E.; TOMLIN, R. P.; PROPPER, R.; DUNCAN, D. W. Ambient Air Concentrations of Pesticides in California. Environmental Science \& Technology, Easton, v. 30, n. 4, p. 1365-1368, May, 1996.

BARAUD, L.; TESSIER, D.; AARON, J. J.; QUISEFIT, J. P.; PINART, J. A multiresidue method for characterization and determination of atmospheric pesticides measured at two French urban and rural sampling sites. Analytical and Bioanalytical Chemistry, Heidelberg, v. 377, n. 7, p. 1148-1152, Dec., 2003.

BATTERMAN, S. A.; CHERNYAK, S. M.; GOUNDEN, Y.; MATOOANE, M.; NAIDOO, R. N. Organochlorine pesticides in ambient air in Durban, South Africa. The Science of the Total Environment, Amsterdam, v. 397, n. 1, p. 119-130, Jul., 2008.

BRASIL. Lein. 7.802, de 11 de julho de 1989. Dispõe sobre a pesquisa, a experimentação, a produção, a embalageme rotulagem, o transporte, o armazenamento, a comercialização, a propaganda comercial, a utilização, a importação, a exportação, o destino final dos resíduos e embalagens, o registro, a classificação, o controle, a inspeção e a fiscalização de agrotóxicos, seus componentes e afins, e dá outras providências. 
Diário Oficial da União; Poder Executivo, de 12 de julho de 1989.

BRASIL. Lein. 9.974, de 06 de junho de 2000. Altera a Lei $n^{\circ} 7.802$, de 11 de julho de 1989, que dispõe sobre a pesquisa, a experimentação, a produção, a embalageme rotulagem, o transporte, o armazenamento, a comercialização, a propaganda comercial, a utilização, a importação, a exportação, o destino final dos resíduos e embalagens, $\mathrm{o}$ registro, a classificação, o controle, a inspeção e a fiscalização de agrotóxicos, seus componentes e afins, e dá outras providências. Diário Oficial da União;Poder Executivo, de 07 de junho de 2000.

BRASIL. Decreto n. 4.074, de 04 de janeiro de 2002. Regulamenta a Lei no 7.802, de 11 de julho de 1989, que dispõe sobre a pesquisa, a experimentação, a produção, a embalagem e rotulagem, o transporte, o armazenamento, a comercialização, a propaganda comercial, a utilização, a importação, a exportação, o destino final dos resíduos e embalagens, o registro, a classificação, o controle, a inspeção e a fiscalização de agrotóxicos, seus componentes eafins, e dá outras providências. Diário Oficial da União; Poder Executivo, de 08 de janeiro de 2002.

BRASIL. Decreto n. 5.549, de 22 de setembro de 2005. Dá nova redação e revoga dispositivos do Decreto no 4.074, de 4 de janeiro de 2002, que regulamenta a Leino 7.802, de 11 de julho de 1989, que dispõe sobre a pesquisa, a experimentação, a produção, a embalageme rotulagem, o transporte, o armazenamento, a comercialização, a propaganda comercial, a utilização, a importação, a exportação, o destino final dos resíduos e embalagens, o registro, a classificação, o controle, a inspeção e a fiscalização de agrotóxicos, seus componentes e afins. Diário Oficial da União; Poder Executivo, de 23 de setembro de 2005.

BRASIL. Decreto n. 5.981, de 06 de dezembro de 2006. Dá nova redação e inclui dispositivos ao Decreto no 4.074, de 4 de janeiro de 2002, que regulamenta a Lei no 7.802, de 11 de julho de 1989, que dispõe sobre a pesquisa, a experimentação, a produção, a embalageme rotulagem, o transporte, o armazenamento, a comercialização, a propaganda comercial, a utilização, a importação, a exportação, o destino final dos resíduos e embalagens, o registro, a classificação, o controle, a inspeção e a fiscalização de agrotóxicos, seus componentes e afins. Diário Oficial da União; Poder Executivo, de 07 de dezembro de 2006.

BRIAND, O.; BERTRAND, F; SEUX, R.; MILLET, M. Comparison of different sampling techniques for the evaluation of pesticide spray drift in apple orchards. The 
Science of the Total Environment, Amsterdam, v. 288, n. 3, p. 199-213, Apr., 2002.

BRIAND, O.; MILLET, M.; BERTRAND, F.; CLEMENT, M.; SEUX, R.; Assessing the transfer of pesticides to the atmosphere during and after application. Dvelopment of a multiresidue method using adsorption on Tenax and thermal desorption-GC/MS. Analytical and Bioanalytical Chemistry, Heidelberg, v. 374, n. 5, 848-857, Nov., 2002.

BUEHLER, S.; BASU, I.; HITES, R. A. AComparison of PAH, PCB, and pesticide concentrations in air at two rural sites on Lake Superior. Environmental Science \& Technology, Easton, v. 35, n. 12, p. 2417-2422, Jun., 2001.

CESSNA, A. J.; WAITE, D. T.; KERR, L. A.; GROVER, R. Duplicate sampling reproducibility of atmospheric residues of herbicides for paired pan and high-volume air samplers. Chemosphere, Oxford, v. 40, n. 8, p. 795-802, Apr., 2000.

COHEN, S.Z.; WAUCHOPE, R. D.; KLEIN, A. W.; EADSFORTH, C. V.; GRANEY, R. Offsite transport of pesticides in water mathematical models of pesticide leaching and runoff. Pure and Applied Chemistry, Oxford, v. 67, n. 12, p. 2109-2148, Dec., 1995.

COSCOLLÀ, C.; YUSÀ, V.; MARTÍ, P.; PASTOR, A. Analysis of currently used pesticides in fine airborne particulate matter (PM2.5) by pressurized liquid extraction and liquid chromatography-tandemmass spectrometry. Journal of Chromatography A, Amsterdam; New York, v. 1200, n. 2, p. 100-107, Jul., 2008.

DOBSON, R.; SCHEYER, A.; RIZET,A. L.; MIRABEL, O.; MILLET, M. Comparison of the efficiencies of different types of adsorbents at trapping currently used pesticides in the gaseous phase using the technique of high-volume sampling. Analytical and Bioanalytical Chemistry, Springer, v. 386, n. 6, p. 1781-1789, Nov., 2006.

DUYZER, J. Pesticide concentrations in air and precipitation in the Netherlands. Journal of Environmental Monitoring, Cambridge, v. 5, n. 4, 77N-80N, Aug., 2003.

ELFLEIN,L.; BERGER-PREISS, E.; LEVSEN, K.; WUNSCH, G. Development of a gas chromatography-mass spectrometry method for the determination of household insecticides in indoor air. Journal of Chromatography, A, Amsterdam; New York, v. 985, n. 1, p. 147-157, Jan., 2003. 
EMPRESABRASILEIRADE PESQUISAAGROPECUÁRIA. Circular técnica 74: Inseticidas registrados para a cultura do arroz e análise de parâmetros indicadores de seu comportamento no ambiente. Santo Antônio de Goiás, 2005. 4p.

FALCONER, K. Pesticide environmental indicators and environmental policy. Journal of Environmental Management, London, v. 65, n. 3, p. 285-300, Jul., 2002.

FERRARI, F;; TREVISAN, M.; CAPRI, E. Predicting and Measuring Environmental Concentration of Pesticides in Air after Soil Application. Journal of Environmental Quality, Madison, v. 32, n.5, p. 1623-1633, Set/Out, 2003.

FILIZOLA, H. F.; FERRACINI, V. L.; SANS, L. M. A.; GOMES, M. A. F.; FERREIRA, C. J.A. Monitoramento e avaliação do risco de contaminação por pesticidas emágua superficial e subterrânea na região de Guaíra. Pesquisa Agropecuária Brasileira, Brasilia, v. 37, n. 5, p. 659-667, May, 2002.

FONTANALS, N.; MARCÉ, R. M.; BORRULL, F. New materials in sorptive extraction techniques for polar compounds. Journal of Chromatography,A, v. 1152, n.1-2, 14-31, Jun., 2007.

GIL, Y.; SINFORT, C. Emission of pesticides to the air during sprayer application: A bibliographic review. Atmospheric Environment, Oxford, v.39, n. 28, p. 5183-5193, Sep., 2005.

GOUIN, T.; HARNER, T.; BLANCHARD, P.; MACKAY, D. Passive and active air samplers as complementary methods for investigating Persistent Organic Pollutants in the Great Lakes Basin. Environmental Science \& Technology, Easton, v. 39, n. 23, p. 9115-9122, Dec., 2005.

GOUIN, T.; WANIA, F; RUEPERT, C.; CASTILHO, L. E. Field Testing Passive Air Samplers for Current Use Pesticides in a Tropical Environment. Environmental Science \& Technology, Easton, v. 42, n. 17, p. 6625-6630, Sep., 2008.

HAPEMAN, C. J.; McCONNELL, L. L.; RICE, C. P.; SADEGHI, A. M.; SCHMIDT, W. F.; McCARTY, G. W.; STARR, J. L.; RICE, P. J.;ANGIER, J. T.; HARMAN-FETCHO, J.A. Current United States Department of Agricultureagricultural research service research on understanding agrochemical fate and transport to 
prevent and mitigate adverse environmental impacts. Pest Management Science, West Sussex, v. 59, n. 6-7, p. 681-690, Jun-Jul., 2003.

HARNER, T.; BARTKOW, M.; HOLOUBEK, I.; KLÁNOVA, J.; WANIA, F.; GIOIA, R.; MOECKEL, C.; SWEETMAN, A. J.; JONES, K. C. Passive air sampling for persistent organic pollutants: Introductory remarks to the special issue.

Environmental Pollution, Barking, v. 144, n. 2, 361-364, Nov., 2006.

HARRAD, S., MAO, H. Atmospheric PCBs and organochlorine pesticides in Birmingham, UK: concentrations, sources, temporal and seasonal trends. Atmospheric Environment, Oxford, v. 38, n. 10, p. 1437-1445, Mar., 2004.

HERCEG, R. S.; KRAUTHACKER, B. Comparison of ambient air levels of PCBs and organochlorine pesticides at two sites in Zagreb, Croatia. Bulletin of Environmental Contamination and Toxicology, New York, v. 71, n. 5, p. 1034-1041, Nov., 2003.

JAWARD, F. M.; FARRAR, N. J.; HARNER, T.; SWEETMAN, A. J.; JONES, K. C. Passive air sampling of PCBs, PBDEs, and organochlorine pesticides across Europe. Environmental Science \& Technology, Easton, v. 38, n. 1, p. 34-41, Jan., 2004.

LAMMEL, G; GHIM, Y.; GRADOS, A.; GAO, H.; HÜHNERFUSS, H.; LOHMANN, R. Levels of persistent organic pollutants in air in China and over the Yellow Sea. Atmospheric Environment, Oxford, v. 41, n. 3, p. 452-464, Jan., 2007.

LEPRI, L.; DESIDERE, P.; CINI, R.; MASI, F.; VAN ERK, M. S. Transport of organochlorine pesticides across the air/sea interface during the aerosol process. Analytica Chimica Acta, Amsterdam, v. 317, n. 1-3, p. 149-160, Dec., 1995.

LEWIS, R. G; MACLEOD, K. E. Portable sampler for pesticides and semivolatile industrial organic chemicals in air. Analytical Chemistry, Washington, v. 54, n. 2, p. 310-315, Feb., 1982.

MAJEWSKI, M.; FOREMAN, W.; GOOLSBY, D.; NKAHAKI, N. Airborne Pesticide Residues along the Mississippi River. EnvironmentalScience \& Technology, Easton, v. 32, n. 23, p. 3689, Dec., 1998.

MILLET, M.;WORTHAM, H.; SANUSI, A.; MIRABEL, P.A multiresidue method for determination of trace levels of pesticides. Archives of Environmental 
Contamination and Toxicology, New York, v. 31, n. 4, p. 543-556, Nov., 1996.

MOTELAY-MASSEI, A.; HARNER, T.; SHOEIB, M.; DIAMOND, M.; STERN, G; ROSENBERG B. Using passive air samplers to assess urban-rural trends for persistent organic pollutants and polycyclic aromatic hydrocarbons. 2. Seasonal trends for PAHs, PCBs, and organochlorine pesticides. Environmental Science \& Technology. Easton, v. 39, n. 15, 5763-5773, Aug, 2005.

MURAYAMA, H.; MUKAI, H.; MITOBE, H.; MORIYAMA, N. Simple method for determining trace pesticides in air using extraction disks. Analytical Sciences, Tokyo, v. 16, p. 257-263, Mar., 2000.

PECK, A. M.; HORNBUCKLE, K. C. Gas-phase concentrations of current-use pesticides in Iowa. Environmental Science \& Technology, Easton, v. 39, n. 9, p. 2952-2959, May, 2005.

POZO, K.; HARNER, T.; SHOEIB, M.;URRUTIA, R.;BARRA, R.;PARRA, O.; FOCARDI, S. Passive-Sampler derived air concentrations of Persistent Organic Pollutants on a North-South transect in Chile. Environmental Science \& Technology, Easton, v. 38, n. 24, p. 6529-6537, Dec., 2004.

RAINA, R.; SUN, L. Trace level determination of selected organophosphorus pesticides and their degradation products in environmental air samples by liquid chromatography-positive ion electrospray tandem mass spectrometry. Journal of Environmental Science and Health, Part B, New York, v. 43, n. 4, p. 323-332, May, 2008.

RANGEL, Cássia de Fátima. Monitoramento de agrotóxicos em águas brutas e tratadas destinadas ao consumo humano, utilizandométodo multi-resíduo por EFS/CG-EM. 130 p. Dissertação de Mestrado - Escola Nacional de Saúde Pública Sergio Arouca, Rio de Janeiro, 2008.

RIBANI, M.; BOTTOLI, C. B. G.; COLLINS, C. H.; JARDIM, I. C. S. F.; MELO, L. F. C. Validação em métodos cromatográficos e eletroforéticos. Química Nova, São Paulo, v. 27, n. 5, p. 771-780, Set/Out, 2004.

SANUSI, A.; MILLET, M.; MIRABEL, P.; WORTHAM, H. Gas-particle partitioning of pesticides in atmospheric samples. Atmospheric Environment, 
Oxford, v. 33, n. 29, p. 4941-4951, Dec., 1999.

SANUSI, A.; MILLET, M.; MIRABEL, PH.; WORTHAM, H. Comparison of atmospheric pesticide concentrations measured at three sampling sites: local, regional and long-range transport. The Science of the Total Environment, Amsterdam, v. 263, n. 1, p. 263-277, Dec., 2000.

SAURET, N.; WORTHAM, H.; PUTAUD, JEAN-PHILIPE, MIRABEL, P. Study of the effects of environmental parameters on the gas/ particle partitioning of current-use pesticides in urban air. Atmospheric Environment, Oxford, v. 42, n. 3, p. 544-553, Jan., 2008.

SCHEYER, A.; GRAEFF, C.; MORVILLE, S.; MIRABEL, P.; MILLET, M. Analysis of some organochlorine pesticides in an urban atmosphere (Strasbourg, east of France). Chemosphere, Oxford, v. 58, n. 11, p. 1517-1524, Mar., 2005.

SCHEYER, A.; MORVILLE, S.; MIRABEL, P.; MILLET, M. Variability of atmospheric pesticide concentrations between urban and rural areas during intensive pesticide application. Atmospheric Environment, Oxford, v. 41, n. 17, p. 36043618, Jun., 2007.

SOFUOGLU,A.; ODABASI, M.; TASDEMIR,Y.; KHALILI, N. R.; HOLSEN, T. M. Temperature dependence of gas-phase polycyclic aromatic hydrocarbon and organochlorine pesticide concentrations in Chicago air. Atmospheric Environment, Oxford, v. 35, n. 36, p. 6503-6510, Dec., 2001.

SPADOTTO, C. A.; GOMES, M. A. F.; LUCHINI, L. C.; ANDRÉA, M. M. Monitoramento do risco ambiental de agrotóxicos: princípios e recomendações. Jaguariúna: Embrapa Meio Ambiente, 2004. 29 p. (Documentos, 42).

UNSWORTH, J. B.; WAUCHOPE, R. D.; KLEIN, A. W.; DORN, E.; ZEEH, B.; YEH, S. M.;AKERBLOM, M.; RACKE, K. D.; RUBIN, B. Significance of the long-range transport of pesticides in the atmosphere: technical report. Pure and Applied Chemistry, Oxford, v. 71, n. 7, p. 1359-1383, Jul., 1999.

VAN DEN BERG, F.; KUBIAK, R.; BENJEY, W. G.; MAJEWSKI, M. S.; YATES, S. R.; REEVES, G. L.; SMELT, J. H.; VAN DER LINDEN, A. M. A. Emission of pesticides into the air. Water, Air and Soil Pollution, Springer, v. 115, 
n. 1-4, p. 195-218, Oct., 1999.

VAN DIJK, H. F. G; GUICHERIT, R. Atmospheric dispersion of current-use pesticides: review of the evidence frommonitoring studies. Water, airand soil pollution, Springer, v. 115, n. 1-4, p. 21-70, Oct., 1999.

VAN JAARSVELD, J. A.; VAN PUL, W.A. J. Modelling of atmospheric transport and deposition of pesticides. Water,Air and Soil Pollution, Springer, v. 115, n. 1-4, p. 167-182, Oct., 1999.

VAN PUL, W.A. J.; BIDLEMAN, T. F; BRORSTROEM-LUNDEN, E.; BUILTJES, P. J. H.; DUTCHAK, S.; DUYZER, J. H.; GRYNING, S-E.; JONES, K. C.; VAN DIJK, H. F. G; VAN JAARSVELD, J. A. Atmospheric transport and deposition of pesticides: an assessment of current knowledge. Water, Air and Soil Pollution, Springer, v. 115, n. 1-4, p. 245-256, Oct., 1999.

VILLA, F; McLEOD, H. Environmental vulnerability indicators for environmental planning and decision-making: guidelines and applications. Environmental Management, New York, v. 29, n. 3, p.335-348, March, 2002.

XU, D.; MO DAN; SONG,Y.; CHAI, Z.; ZHUANG, G Concentration characteristics of extractable organohalogens in PM2.5 and PM10 in Beijing, China. Atmospheric Environment, Oxford, v. 39, n. 22, p. 4119-4128, Jul., 2005.

ZHANG, G.; CHAKRABORTY, P.; JUN LI, SAMPATHKUMAR, P.; BALASUBRAMANIAN, T.; KATHIRESAN, K.; TAKAHASHI, S.; SUBRAMANIAN, A.; TANABE, S.; JONES, K. C. Passive atmospheric sampling of organochlorine pesticides, polychlorinated biphenyls, and polybrominated diphenyl ethers in urban, rural, and wetland sites along the coastal length of India. Environmental Science \& Technology, Easton, v. 42, n. 22, p. 8218-8223, Nov, 2008.

WAITE, D. T.; CESSNA, A. J.; GROVER, R.; KERR, L. A.; SNIHURA, A. D. Environmental concentrations of agricultural herbicides in Saskatchewan, Canada: bromoxynil, dicamba, diclofop, MCPA, and trifluralin. Journal of Environmental Quality, Madison, v. 33, n. 5, p. 1616-1628, Sep/Oct, 2004.

WAITE, D.T.;BAILEY, P.; SPROULL, J. F.; QUIRING, D. V.; CHAU, D. F.; 
BAILEY, J.; CESSNA, A. J. Atmospheric concentrations and dry and wet deposits of some herbicides currently used on the Canadian Prairies. Chemosphere, Oxford, v. 58, n. 6, p. 693-703, Feb, 2005.

WHITE,L.M.;ERNST, W.R.; JULIEN, G; GARRON, C.; LEGER, M. Ambient air concentrations of pesticides used in potato cultivation in Prince Edward Island, Canada. Pest Management Science, West Sussex, v. 62, n. 2, p. 126-136, Feb, 2006.

YANG, Y.; LI, D.; MU, D. Levels, seasonal variations and sources of organochlorine pesticides in ambient air of Guangzhou, China. Atmospheric Environment, Oxford, v. 42, n. 4, p. 677-687, Feb., 2008.

YAO, Y.; TUDURI, L.; HARNER, T.; BLANCHARD, P.; WAITE, D.; POISSANT, L.; MURPHY, C.; BELZER, W.;AULAGNIER, F.; LI, YI-FAN; SVERKO, E.

Spatial and temporal distribution of pesticide air concentrations In Canadian agricultural regions. Atmospheric Environment, Oxford,v. 40, n. 23, 4339-4351, Jul., 2006.

YUSA, V.; COSCOLLA, C.; MELLOUKI, W.; PASTOR, A.; DE LA GUARDIA, M. Sampling and analysis of pesticides in ambient air. Journal of Chromatography, A, Amsterdam, v. 1216, n. 15, p. 2972-2983, Apr., 2009.

\section{TItLE: PESTICIDE RESIDUES ANALYSIS IN AMBIENT AIR}

\section{ABSTRACT}

Identifying the distribution and levels of pesticides in ambient air can contribute to a better knowledge of the risk associated with these compounds to human health. Taking into account the necessity of efficiently measuring pesticides in this matrix, this article briefly discusses the methods employed, including both active and passive air sampling, pesticides analysis, as well as the procedures that have been implemented formethod validation. Although analytical efforts to obtain reliable results have been demonstrated in the literature, further attempts are required to improve the sampling procedures and analytical methods for determining pesticides in ambient air.

KEYWords: Pesticides; Ambient Air; Sampling and Analysis Procedures. 
Tabela 1. Descrição de estudos que determinam pesticidas em ar atmosférico.

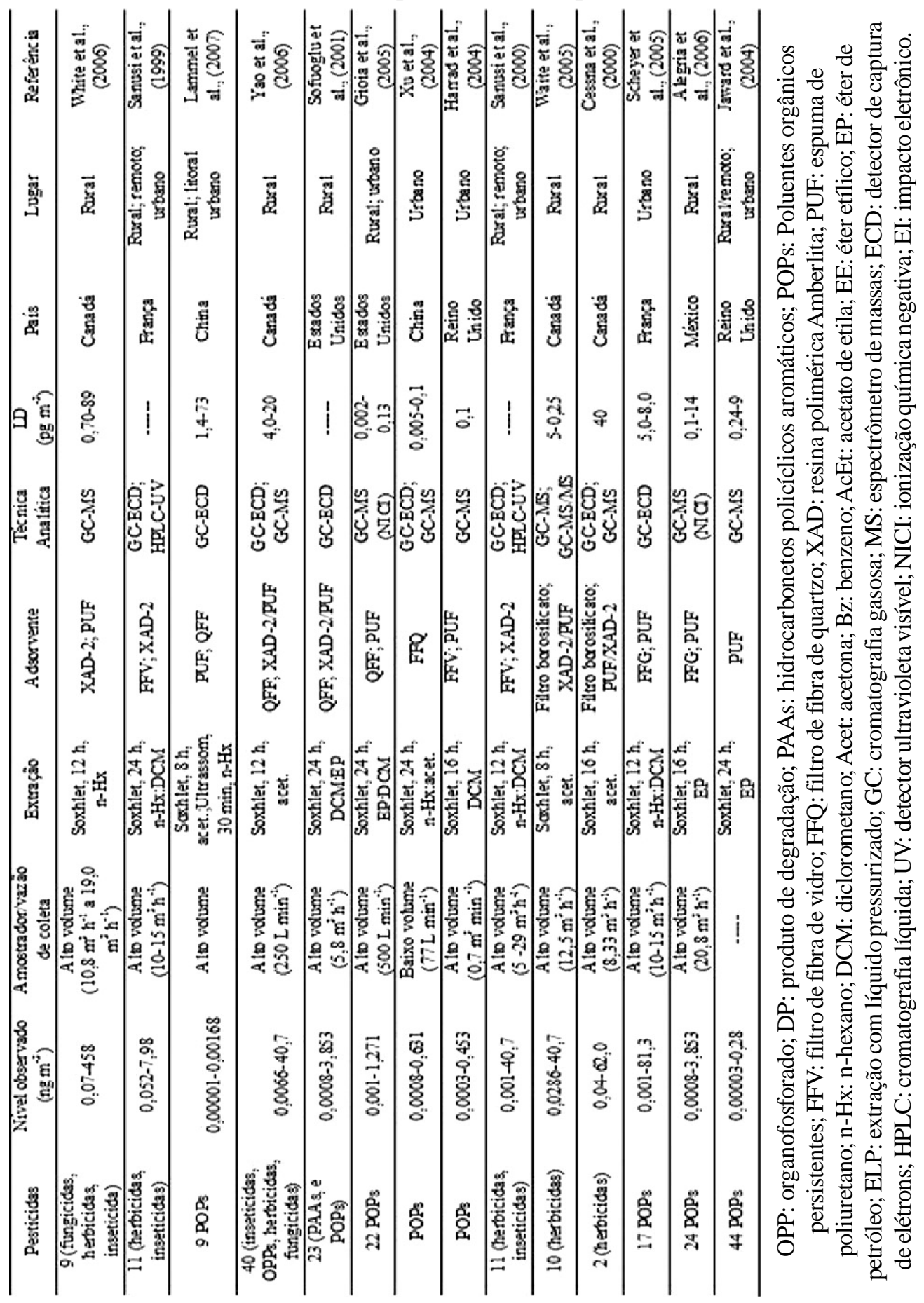


Tabela 1. Continuação

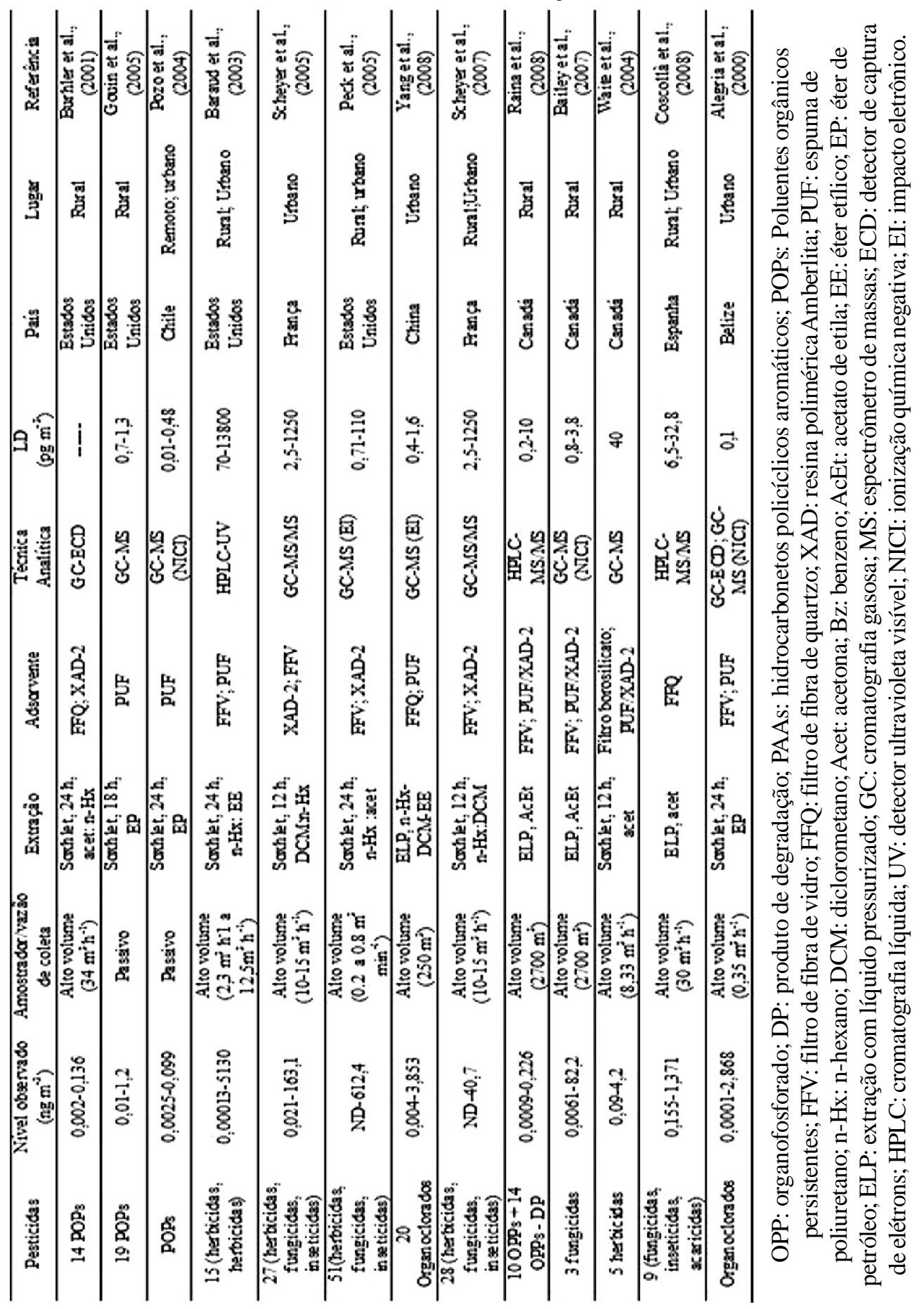


Tabela 1. Continuação

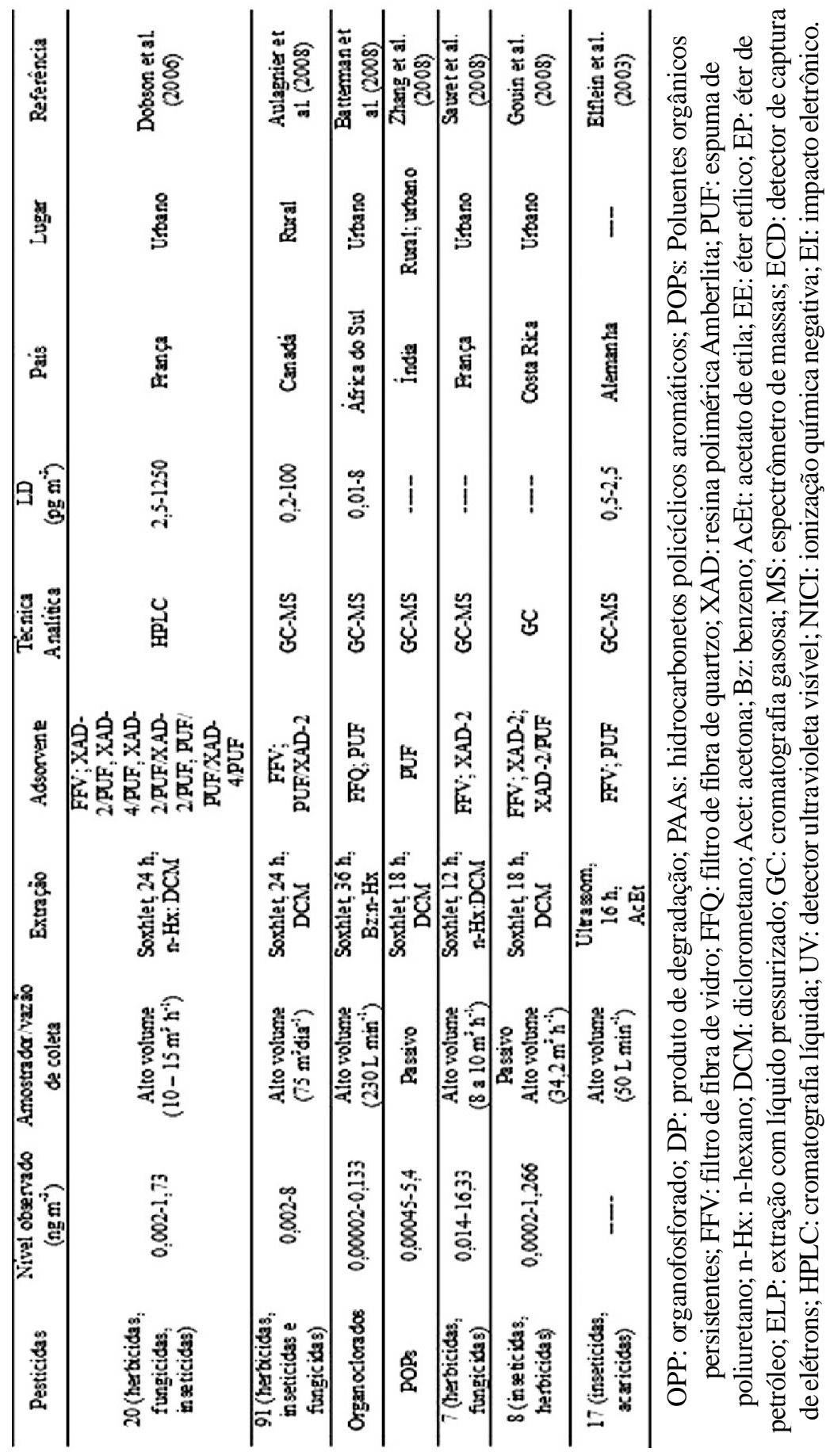

